\title{
Realization of a flat-band superprism on-chip from parallelogram lattice photonic crystals
}

\author{
Jeremy Upham ${ }^{1, *}$, Boshen GaO ${ }^{2}$, Liam O’FaOlain ${ }^{3,4,5}$, Zhimin Shi ${ }^{6}$, Sebastian A. Schulz ${ }^{1,5}$, AND \\ ROBERT W. BOYD ${ }^{1,2}$ \\ ${ }^{1}$ Department of Physics and School of Electrical Engineering and Computer Science, University of Ottawa, Ottawa, K1N 6N5, Canada \\ ${ }^{2}$ The Institute of Optics, University of Rochester, Rochester, New York 14627, USA \\ ${ }^{3}$ Centre for Advanced Photonics and Process Analysis, Cork Institute of Technology,Cork, Ireland \\ ${ }^{4}$ Tyndall, National Institute, Cork, Ireland \\ ${ }^{5}$ SUPA, School of Physics and Astronomy, University of St Andrews, St Andrews, Scotland, UK \\ ${ }^{6}$ Department of Physics, University of South Florida, Tampa, USA \\ *Corresponding author: jupham@uottawa.ca
}

Compiled November 22, 2018

By optimizing the dispersion curve of a parallelogrambased 2D photonic crystal superprism for constant angular group velocity dispersion over a broad bandwidth, we designed a device capable of experimentally demonstrating linear dispersion from 1500 to $1600 \mathrm{~nm}$ with clear separation of as many as 8 channels, while maintaining a compact footprint. () 2018 Optical Society of America

http://dx.doi.org/10.1364/ao.XX.XXXXXX

Separation of an incident broadband signal into distinct spectral channels is a key requirement for spectroscopy and wavelength division multiplexing. However such dispersive elements must achieve a large angular dispersion over the entire bandwidth of interest, which often required a device with a large footprint [1], which is a problem for on-chip devices. For instance, solutions based on arrayed waveguide gratings (AWG) or échelle gratings do exist, yet their footprints are still large [2]. Designs providing greater dispersion engineering are required for the development of practical on-chip spectrometers and demultiplexers. By providing wavelength-order spatial control over optical modes, photonic crystals offer a path to further reduction in the device size, and thus better device density on-chip, by taking advantage of the dispersion engineering to exercise the superprism effect [3].

Superprism-based photonic crystal $(\mathrm{PhC})$ devices engineer the optical mode within the PhC to apply a large angular dispersion to the group velocity, creating greater spatial separation of co-incident light of distinct frequencies than is possible by conventional material dispersion. Superprism devices can better isolate wavelengths to single spatial channels, spectrally filtering light then sending it on to the subsequent optical components on the chip. Therefore PhC superprisms offer significant potential for miniaturized on-chip spectrometers and demultiplexers.

Several works have focused on maximizing the angular group dispersion (AGD) in PhC superprisms, which is to say the difference in angular dispersion between two coincident beams of distinct frequency, so as to achieve the better channel separation in a smaller device footprint [4-6], while addressing issues such as channel crosstalk[7,8]. However, increasing the AGD tends to reduce the operable bandwidth and to produce a non-uniform AGD, meaning that the difference in dispersion angle is not linear with the difference in frequency over the working bandwidth of the device. Accounting for this non-uniformity requires increased design complexity and non-uniform channel spacings. In this work we thus focus on realizing a device that prioritizes a large but near constant AGD over a broad bandwidth, by optimizing a new figure of merit: the angular-group-dispersionbandwidth product (AGDBP) [9]. This includes fabricating a practical superprism PhC device made to maximize the AGDBP, characterizing its performance and comparing it to the benchmark of equivalent nanophotonic systems.

To illustrate how AGD is interpreted in the design of the PhC, we calculate the equi-frequency contours (EFC)[4] in $k$-space for light crossing from a dispersion-free, bulk material into a standard square lattice PhC (Fig. 1a). These contours are obtained by calculating the band structure of the $\mathrm{PhC}$ by the plane wave expansion method[10]. Consider co-incident beams of light traveling from a bulk medium into the PhC. Their refracted beams must conserve their $k$-vector component parallel to the interface and their frequency, which is sufficient to determine the new $k$-vectors. Note that if the EFCs are not circular (meaning that the material is anisotropic), then the $k$-vector does not necessarily normal to the EFC.

As group velocity is the gradient of the frequency in reciprocal space $\left(v_{g}=\nabla_{k} \omega(k)\right)$, it will always point normal to the EFC. The angle of the group velocity vector is denoted by $\beta$. We can then describe the AGD, $q$, as the change of $\beta$ as a function of $\omega$ for a given incident angle $\alpha_{\text {inc }}$ as:

$$
q\left(\alpha_{\mathrm{inc}}, \omega\right)=\left.\frac{\delta \beta}{\delta \omega}\right|_{\alpha_{\mathrm{inc}}} .
$$

Therefore a constant, non-zero $q$ over a given frequency range would indicate a linear relationship between frequency and dispersion angle over that bandwidth. In practice, the angular 

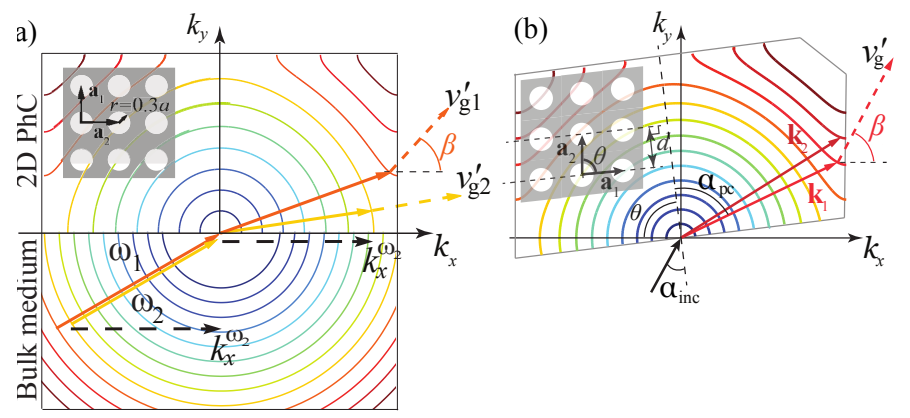

Fig. 1. Equi-frequency contours (EFCs) and representative wavevectors for PhC structures. (a) Light is incident on a square-lattice PhC from a bulk, dispersion-free material. $\beta$ is the angle between the $k_{x}$ axis and the group velocity vector. (b) For a parallelogram lattice $\mathrm{PhC}$, strong anisotropy leads to EFCs that deviate from perfect circles. Thus a small change in frequency leads to a large change in the group velocity direction. The design of superprism devices entails optimizing the variation of $\beta$ with frequency.

group dispersion bandwidth product is thus described as follows:

$$
P\left(\omega_{0}\right)=q\left(\omega_{0}\right) \Delta \omega
$$

where $q\left(\omega_{0}\right)$ is the AGD at a central frequency $\omega_{0}$ and $\Delta \omega$ is the frequency bandwidth in the vicinity of $\omega_{0}$ within which the AGD varies less than $5 \%$ from $q\left(\omega_{0}\right)$. As motivated here and in previous work[9], we maintain that it is the AGDBP $\left(P\left(\omega_{0}\right)\right)$ that ought to be maximized when optimizing the PhC lattice for a practical superprism device.

To maximize both the AGD and practical bandwidth, we consider that hexagonal lattices provide high wavelengthdependent angular dispersion [7], while the EFCs of the square lattice (see again Fig. 1) can be flat over a broad range of wave vectors, suggesting that the AGD of light dispersed along those wave vectors would be close to constant. Therefore, we attempt to hybridize these lattices by optimizing the geometry of a parallelogram lattice $\mathrm{PhC}$. Specifically, we adjust the angle $\theta$ between $a_{1}$ and $a_{2}$ as well as the distance $d$ between two rows (see Fig. 1) and observing how this changes the EFCs and consequently the possible AGDBP. Parameters such as lattice constant and effective index of the slab are selected for practical devices fabricated from silicon and operating for wavelengths near $1550 \mathrm{~nm}$, while the hole radius $r$ is held constant at $0.3 a_{1}$. Sweeping through this parameter space alters the curvature of the EFCs of the lattices (compare Fig. 1 (a) and (b)), revealing the highest AGDBP for incident angle $\alpha_{\text {inc }}$. This optimization leads to the selection of a PhC lattice with parameters: $a_{1}=330 \mathrm{~nm}, a_{2}=297 \mathrm{~nm}$, $d=0.9 a_{1}$ and $\theta=88^{\circ}$ for fabrication, as it shows an optimum $\operatorname{AGDBP}\left(P_{\max }\right)$ of 0.8548 rad for $\alpha_{\text {inc }}=33^{\circ}$.

Another factor to consider for practical superprism-based devices is crosstalk between channels at the output. As beams of different wavelengths are diverted by dispersion in the superprism, they also broaden due to the anomalous diffraction. To negate this diffraction, the light is made to propagate a set distance (here referred to as the pre-conditioning region) in the slab of the silicon chip before reaching the PhC interface. Light broadened by ordinary diffraction in the slab mode is refocused by anomalous diffraction in the superprism, negating each other at the channel outputs [6] and thus reducing crosstalk (see Fig. 2).

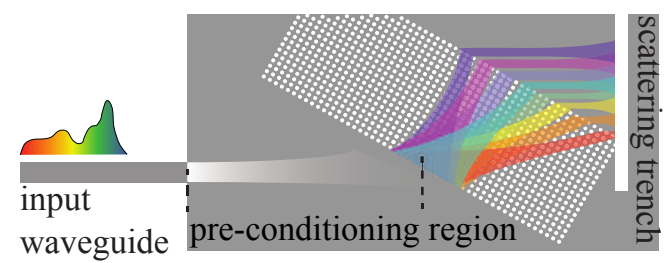

Fig. 2. Schematic of the overall superprism structure, including the conventional diffraction within the preconditioning region, which compensates for the anomalous diffraction of the superprism. The resulting spatially seperate channels at output then scatter off a trench for visual confirmation of dispersion.

Based on complete designs with optimized AGDBP and appropriate pre-conditioning regions proposed in ref [9], devices were fabricated in a $220 \mathrm{~nm}$ thick silicon-on-insulator platform. The pattern was defined in ZEP520A resist using electron beam lithography (Raith Pioneer, 30kV). After development, the sample pattern was transfered using a reactive ion etch with a CHF3/SF6 gas mixture. Subsequently the underlying buried oxide was removed by a hydrofluoric acid etch, to create a membrane $\mathrm{PhC}$ superprism with a symmetric index profile and maximized refractive index contrast.

The fabricated device consisted of a single-mode tapered ridge waveguide followed by a 148 um pre-conditioning region, then a PhC composed of $820 \times 140$ air holes. Two designs of the superprism output were fabricated. The first was a straight, scattering trench normal to the path of the light after the superprism to observe to observe the device performance as the wavelength continuously tunes over the entire bandwidth. The second replaced the scattering trench with 8 single-mode waveguides to determine how well the demultiplexed light could be coupled to isolated channels.

The sample was characterized using light from a tunable laser (Santec TSL-510) coupled to the sample via lensed fibre coupling. A manual fibre polarization controller is used to ensure that the input is TE polarized. After propagating through the preconditioning region and the $\mathrm{PhC}$ superprism, the light is incident onto either the scattering edge, or the collection waveguides (leading to their own scattering edge) and the vertically scattered light is collected using a microscope objective and infrared camera.
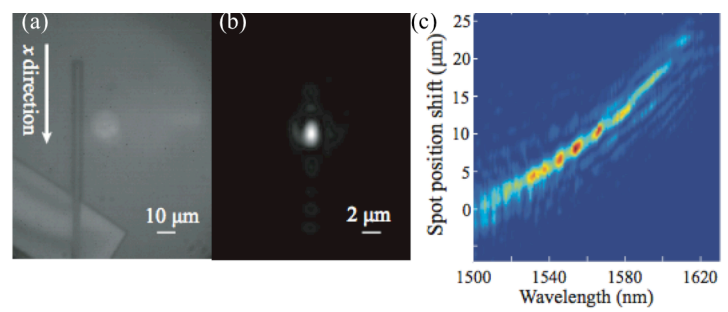

Fig. 3. a) Image of the scattering trench and part of the PhC region (b), light scattered from the trench; (c), spot position along the $\mathrm{x}$ direction versus input wavelength.

Figure 3 shows the experimental results for the scattering edge output (Fig. 3a). The small spot size (Fig. 3b) confirms that the pre-conditioned light experienced negative diffraction in the 
$\mathrm{PhC}$ area. The superprism achieves a near linear angular displacement of the input beam as a function of input wavelength, resulting in a continuous (zero-channel separation) coverage of the full spectral range $(1500 \mathrm{~nm}<\lambda<1600 \mathrm{~nm})$ considered (Fig. $3 c)$. Given a spot position vs wavelength slope of approximately $0.2 \mathrm{um} / \mathrm{nm}$ and an average spot size of $3 \mathrm{um}$, this suggests that such a device could reasonably achieve channel separation of about $15-16 \mathrm{~nm}$. The angular displacement is $0.82 \mathrm{rad}$, which is consistent with the design value of $P_{\max }=0.85 \mathrm{rad}$.

(a)

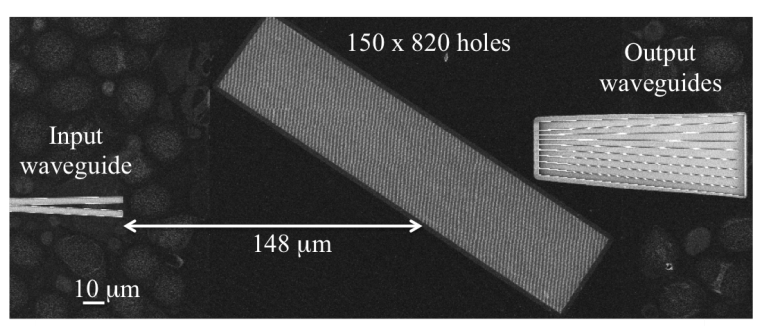

(b)

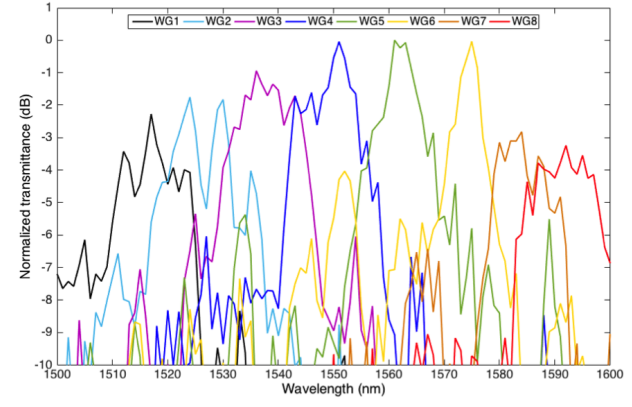

Fig. 4. (a) SEM image of an 8-channel superprism spectrometer. (b), the spectral flux transmission measured at the end of each of the eight single mode output waveguides.

While the scattering edge demonstrates the linear dispersion of our superprism, on-chip demultiplexing and spectroscopy applications require that the outputs be coupled to waveguides and sent to subsequent components on the chip. We have thus replaced the scattering edge with 8 single-mode, ridge waveguides, designed to have a uniform channel spacing of $12 \mathrm{~nm}$ (Fig. $4 a)$. Because they are single mode, these waveguides could be directly coupled on to other on-chip components or to photodiodes to provide electric readouts. Fig. $4 \mathrm{~b}$ shows the optical outputs from this 8 channel superprism spectrometer. The measured channel spacing is roughly $12 \mathrm{~nm}$ with the observed peak transmission at the scattering output of the waveguides varying by $5 \mathrm{~dB}$ between the channels and a typical channel crosstalk of 3-4 dB. Note that previous simulations of this 8 channel spectrometer design predicted peak transmission variation of $4 \mathrm{~dB}$ and typical cross talk of about $10 \mathrm{~dB}$ [9], which are reasonable agreement between FDTD simulations and experimental measurement.

We attribute the difference between the observed and expected performance to variations in the parameters of the fabricated device (e.g. the width of the collection waveguides) and to the fact that the effective index approximation was used to design the device. Further design refinement to better define the preconditioning section and the PhC parameters, perhaps by using $3 \mathrm{D}$ simulations, could improve performance.

To better evaluate the advantages and disadvantages of our design, we compare our device to some representative examples of superprisms and other on-chip compact spectral filter systems. Several characteristics of interest are listed in Table 1: the functional spectral range, the spectral resolution, the number of resolvable channels, the footprint of the device and, where relevant, the crosstalk between channels.

First, optimizing for a maximum AGDBP allowed for a device with more channels and a smaller footprint than comparable superprism designs [6, 7]. The full device in ref. [7] requires long adiabatic broadening of the input waveguides, that are not included in the device footprint, but very likely make the footprint larger than the device in this work. In both of these cases the outcoupling waveguides are several microns wide instead of single-mode, thus achieving lower crosstalk at the expense of the number of channels.

Next we consider on-chip spectroscopy designs, which typically show much higher spectral resolution over a narrower bandwidth. Because optimizing for AGDBP in that particular range could produce a $\mathrm{PhC}$ design with a proportional increase in spectral resolution, we think this approach should be considered for such applications as well. Even without being optimized for the same spectral range, the channel density of our device is higher than for cascaded M-Z devices [11]. The nanocavity array [12] and disordered photonic chip [13] also take advantage of the PhCs wavelength-order spatial control of light to achieve a better channel density, but neither permit the spectrally filtered light to remain on-chip, instead scattering it out of plane. Therefore, while they certainly could be preferred when connecting directly to detectors, they would not be viable for applications where the signal needs to continue on to other on-chip components.

Another way of evaluating our flat-band superprism device is to look at it's potential in the specific application of $25 \mathrm{GHz}$ channel spacing on the standard frequency grid for telecommunications. This would require roughly $0.2 \mathrm{~nm}$ channel spacing between $1530 \mathrm{~nm}$ and $1625 \mathrm{~nm}$ to carry 512 channels, which the AWG in ref [14] was built to achieve. Clearly the device implemented in this paper does not reach this level of channel resolution, but the bandwidth of uniform dispersion is the same so we can estimate the necessary size of a device using the same $\mathrm{PhC}$ design to achieve this spectral resolution. Including the necessary pre-conditioning region and allowing for the channels to be sufficiently spatially separated to have at most $4 \mathrm{~dB}$ crosstalk, we estimate the footprint of such a device would be $9 \mathrm{~mm}$ by $9.5 \mathrm{~mm}$, about half the size of the aformentioned AWG. Note that the AWG is actually a $512 \times 512$ router and thus has additional functionalities, but comparing their sizes suggests that a properly designed superprism could reach similar performance metrics.

In summary, we demonstrated that the AGDBP is a useful figure of merit for the design of practical superprisms, be it for nano-scale spectroscopy, on-chip demultiplexers or compact spectral filters. Our device is the first experimental demonstration of a superprism optimised for a flat-top AGD over a large bandwidth. Optimizing the superprism according to AGDBP, rather than just maximising the AGD, resulted in a large and linear angular dispersion as a function of input wavelength over the complete operating range, here spanning from $1500 \mathrm{~nm}$ to $1600 \mathrm{~nm}$. We measured a maximal angular displacement of 0.82 rad (compared to a theoretically predicted AGDBP value of 0.85 $\mathrm{rad).} \mathrm{As} \mathrm{we} \mathrm{have} \mathrm{demonstrated,} \mathrm{this} \mathrm{scheme} \mathrm{is} \mathrm{well-suited} \mathrm{to}$ coupling to output waveguides and can therefore be integrated with other integrated photonic devices. We have observed a typical channel crosstalk of $4 \mathrm{~dB}$ with $15 \mathrm{~nm}$ channel spacing, compared to the typical simulated value of $10 \mathrm{~dB}$. As on-chip photonic devices become more advanced, small scale yet pre- 
Table 1. Comparison of integrated spectral filtering devices

\begin{tabular}{|c|c|c|c|c|c|}
\hline Design & Spectral range & $\delta \lambda$ & Channels & Footprint & Crosstalk \\
\hline Pre-conditioning superprism [6] & $1530 \sim 1565 \mathrm{~nm}$ & $8 \mathrm{~nm}$ & 4 & $1150 \mu \mathrm{m} \times 90 \mu \mathrm{m}$ & $6.5 \mathrm{~dB}$ \\
\hline Course wave demux superprism [7] & $1480 \sim 1600 \mathrm{~nm}$ & $25 \mathrm{~nm}$ & 4 & $>65 \mu \mathrm{m} \times 42 \mu \mathrm{m}$ & $16-20 \mathrm{~dB}$ \\
\hline Flat-band superprism (this work) & $1510 \sim 1600 \mathrm{~nm}$ & $15 \mathrm{~nm}$ & 8 & $220 \mu \mathrm{m} \times 120 \mu \mathrm{m}$ & $3-4 \mathrm{~dB}$ \\
\hline Cascaded M-Z interferometers [11] & $1549.625 \sim 1550.375 \mathrm{~nm}$ & $42 \mathrm{pm}$ & 18 & $12 \mathrm{~mm}^{2}$ & \\
\hline Nanocavities array [12] & $805 \sim 840 \mathrm{~nm}$ & $0.35 \mathrm{~nm}$ & 100 & $50 \mu \mathrm{m} \times 50 \mu \mathrm{m}$ & $10 \mathrm{~dB}$ \\
\hline Disordered photonic chip [13] & $1500 \sim 1525 \mathrm{~nm}$ & $0.75 \mathrm{~nm}$ & 33 & $25 \mu \mathrm{m} \times 50 \mu \mathrm{m}$ & $30 \mathrm{~dB}$ \\
\hline Arrayed-waveguide grating [14] & $1500 \sim 1600 \mathrm{~nm}$ & $0.2 \mathrm{~nm}$ & 512 & $11 \mathrm{~mm} \times 16 \mathrm{~mm}$ & $4 \mathrm{~dB}$ \\
\hline
\end{tabular}

cise spectrally selective components such as these superprisms could significantly improve functionality. Further investigation into different PhC lattice structures using the ADGDBP may yet reveal designs even better optimized for on-chip dispersive applications.

\section{ACKNOWLEDGMENTS}

The research data supporting this publication can be accessed at https://doi.org/10.17630/03a6aa69-8fd7-441f-a0be4a7bee62c663.

\section{REFERENCES}

1. Z. Shi and R. Boyd, J. Opt. Soc. Am. B (2008).

2. S. Pathak, P. Dumon, D. V. Thourhout, and W. Bogaerts, IEEE Photon. J. 6, 4900109 (2014).

3. H. Kosaka, T. Kawashima, A. Tomita, M. Notomi, T. Tamamura, T. Sato, and S. Kawakami, Phys. Rev. B 58, R10096 (1998).

4. L. Wu, M. Mazilu, T. Karle, and T. F. Krauss, IEE J. Quantum Electron. 38, 915 (2002).

5. A. Lupu, E. .Cassan, S. Laval, L. E. Melhaul, P. Lyan, and J. M. Fedell, Opt. Express 12, 5690 (2004).

6. B. Momeni, J. Huang, M. Soltani, M. Askari, S. Mohammadi, M. Rakhshandehroo, and A. Adibi, Opt. Express 14, 2413- 2422 (2006).

7. D. Bernier, X. L. Roux, A. Lupu, D. Marris-Morini, L. Vivien, and E. Cassan, Opt. Express 16, 17209 (2008).

8. A. Khorshidahmad and A. G. Kirk, Opt. Express 18, 20518 (2010).

9. B. Gao, Z. Shi, and R. Boyd, Opt. Express 23, 6491 (2015).

10. S. G. Johnson and J. D. Joannopoulos, Opt. Express 8, 173 (2001).

11. A. V. Velasco, P. Cheben, P. J. Bock, A. Delâge, J. H. Schmid, J. Lapointe, S. Janz, M. L. Calvo, D.-X. Xu, M. Florjańczyk, and M. Vachon, Opt. Lett 38, 706 (2013).

12. X. Gan, N. Pervez, I. Kymissis, F. Hatami, and D. Englund, APL 100, 231104 (2012).

13. B. Redding, S. F. Liew, R. Sarma, and H. Cao, Nat. Photon. 7, 751 (2013).

14. S. Cheung, T. Su, K. Okamoto, and S. J. B. Yoo, IEEE J. Sel. Top. Quantum Electron. 20, 310 (2014). 


\section{FULL REFERENCES}

1. Z. Shi and R. Boyd, "Slow-light interferometry: practical limitations to spectroscopic performance," J. Opt. Soc. Am. B (2008).

2. S. Pathak, P. Dumon, D. V. Thourhout, and W. Bogaerts, "Comparison of awgs and echelle gratings for wavelength division multiplexing on silicon-on-insulator," IEEE Photon. J. 6, 4900109 (2014).

3. H. Kosaka, T. Kawashima, A. Tomita, M. Notomi, T. Tamamura, T. Sato, and S. Kawakami, "Superprism phenomena in photonic crystals," Phys. Rev. B 58, R10096-R10099 (1998).

4. L. Wu, M. Mazilu, T. Karle, and T. F. Krauss, "Superprism phenomena in planar photonic crystals," IEE J. Quantum Electron. 38, 915 (2002).

5. A. Lupu, E. .Cassan, S. Laval, L. E. Melhaul, P. Lyan, and J. M. Fedell, "Experimental evidence for superprism phenmone in soi photonic crystals," Opt. Express 12, 5690 (2004).

6. B. Momeni, J. Huang, M. Soltani, M. Askari, S. Mohammadi, M. Rakhshandehroo, and A. Adibi, "Compact wavelength demultiplexing using focusing negative index photonic crystal superprisms," Opt. Express 14, 2413-2422 (2006).

7. D. Bernier, X. L. Roux, A. Lupu, D. Marris-Morini, L. Vivien, and E. Cassan, "Compact, low cross-talk cwdm demultiplexer using photonic crystal superprism,", Opt. Express 16, 17209-17214 (2008).

8. A. Khorshidahmad and A. G. Kirk, "Composite superprism photonic crystal demultiplexer: analysis and design," Opt. Express 18, 2051820528 (2010).

9. B. Gao, Z. Shi, and R. Boyd, "Design of flat-band superprism structures for on-chip spectroscopy," Opt. Express 23, 6491-6496 (2015).

10. S. G. Johnson and J. D. Joannopoulos, "Block-iterative frequency-77 domain methods for maxwell's equations in a planewave basis," Opt. Express 8, 173-190 (2001).

11. A. V. Velasco, P. Cheben, P. J. Bock, A. Delâge, J. H. Schmid, J. Lapointe, S. Janz, M. L. Calvo, D.-X. Xu, M. Florjańczyk, and M. Vachon, "High- resolution fourier-transform spectrometer chip with microphotonic silicon spiral waveguides," Opt. Lett 38, 706-708 (2013).

12. X. Gan, N. Pervez, I. Kymissis, F. Hatami, and D. Englund, "A highresolution spectrometer based on a compact planar two dimensional photonic crystal cavity array," APL 100, 231104 (2012).

13. B. Redding, S. F. Liew, R. Sarma, and H. Cao, "Compact spectrometer based on a disordered photonic chip," Nat. Photon. 7, 751 (2013).

14. S. Cheung, T. Su, K. Okamoto, and S. J. B. Yoo, "Ultra-compact silicon photonic 512 × $51225 \mathrm{ghz}$ arrayed waveguide grating router," IEEE J. Sel. Top. Quantum Electron. 20, 310-316 (2014). 